Pacific Journal of Mathematics

THE LIMIT OF A SEQUENCE OF SQUARES IN AN ALGEBRA
NEED NOT BE A SQUARE 


\title{
THE LIMIT OF A SEQUENCE OF SQUARES IN AN ALGEBRA NEED NOT BE A SQUARE
}

\author{
RICHARD ARENS \\ Dedicated to the memory of Professor Henry A. Dye
}

Let $A_{1} \leftarrow A_{2} \leftarrow A_{3} \leftarrow \cdots$ be an inverse system of Banach-algebra homomorphisms, and let $f$ be an element of the limit algebra $A$. Suppose $f$ is the limit of squares, that is,

$$
f_{1} \hookleftarrow f_{2} \hookleftarrow f_{3} \hookleftarrow \cdots \leftrightarrow f,
$$

where each $f_{n}$ has a square root in its algebra $A_{n}$. Does this require that $f$ have a square root in $A$ ? Our object is to show that the answer to this as well as some similarly natural questions is 'no'.

1. A topological reformulation. Let $\Sigma$ be a covering of a topological space $X$. We will say that $X$ has the $\Sigma$-topology if a set $F$ is closed in $X$ if and only if $F \cap S$ is closed for every member $S$ of $\Sigma$ [2].

In what follows, $\Sigma$ will be an increasing sequence of compact subsets of $X$.

Let $P$ be the cartesian product of countably many copies of the unit circle $U(1)$, with the usual compact topology. Let $X_{p}$ be those sequences $x=\left(\lambda_{1}, \lambda_{2}, \lambda_{3}, \ldots\right)$ for which the numbers $\left(\lambda_{n}\right)^{2}$ with $n \geq p$ are all equal to $\left(\lambda_{p}\right)^{2}$. This set is closed in $P$ and hence is compact.

Let $X$ be the union of these compact spaces $X_{n}$. The $X_{n}$ form a covering $\Sigma$ for $X$ and thus define a $\Sigma$-topology for $X$. This $\Sigma$-topology, and not the topology $X$ inherits from $P$, will be meant when we say 'the topology of $X$ '.

Let $x=\left(\lambda_{1}, \lambda_{2}, \lambda_{3}, \ldots\right)$ be a point of $P$. Define $g_{n}(x)$ to be $\lambda_{n}$. Then $g_{n}$ is continuous on $P$ and hence on each $X_{q}$ and hence on $X$.

For $x=\left(\lambda_{1}, \lambda_{2}, \lambda_{3}, \ldots\right)$ in $X$, supposing it to lie in $X_{n}$ define $f(x)$ to have the value $\left(\lambda_{n}\right)^{2}$. On $X_{n}, g_{n}^{2}$ agrees with $f$, so $f$ is continuous on that set. Thus $f$ is continuous on $X$.

Consider a curve $\alpha(t)$ lying in $X_{n}$. If it is continuous relative to the topology $X_{n}$ inherits from $P$, it is continuous as a curve in $X$.

THEOREM 1. $f$ has no continuous square root on $X$.

Proof. Suppose $g$ were a continuous square root of $f$. Then $g(x)=$ $g(y)$ if $x$ and $y$ agree from some point on, because the obvious curve 
from $x$ to $y$ lies in some $X_{n}$. In fact, $f$ is constant on this curve and $g$ is always one of the square roots of that constant, hence constant.

Suppose some directed set $x_{k}$ lies in some $X_{n}$ and converges to a point $y$ there, in the topology $X_{n}$ inherits from $P$. Then it converges in $X$ to $y$. An example is the sequence given by $\left\{x_{k}\right\}$ where $x_{k}$ has $k(-1)$ 's followed by $1,1,1, \ldots$, and $y=(-1,-1,-1, \ldots)$. All these points lie in $X_{1}$. Now $g(1,1,1, \ldots)$ is surely \pm 1 . It will suffice to consider the case where it is +1 . Then $g\left(x_{k}\right)$ is also 1 , and $g(-1,-1,-1, \ldots)$ is 1 because $g$ is continuous on $X_{1}$.

Consider $\alpha(t)=\left(e^{i t}, e^{i t}, e^{i t}, \ldots\right)$. Because it lies in $X_{1}$, it is a curve connecting $(1,1,1, \ldots)$ to $(-1,-1,-1, \ldots)$ as $t$ varies from 0 to $\pi$. Now $g(\alpha(t))= \pm e^{i t}$. For $t=0$, the sign is + , so it must be also for $t=\pi$. Thus $g(-1,-1,-1, \ldots)$ is -1 . This contradicts the earlier finding that this value is +1 . Thus there cannot be a continuous square root of our $f$, proving the theorem.

2. Generalization to powers greater than the second. Let $P$ be as above. Let $H$ be those sequences $x=\left(\lambda_{1}, \lambda_{2}, \lambda_{3}, \ldots\right)$ for which the sequence of numbers $\left(\lambda_{n}\right)^{n}$ ultimately assumes some constant value in $U(1)$. Define $f(x)$ as equal to that limiting value.

Let $H_{n}$ be the subset of $P$ of those $x$ for which that limiting value has already been attained with $\left(\lambda_{n}\right)^{n}$. Note that $f$ has a continuous $n$th root $g_{n}$ on $H_{n}$.

It is natural to ask, does $f$ have a square root $h$ on $P$ ? It does not. We take $\alpha(t)=\exp (i t / n)$. Let $y=\alpha(2 \pi)$, and let $x_{k}$ look like $y$ for the first $k$ places and then have 1's. All these $x_{k}$ and the $y$ lie in $H_{1}$, so $x_{k}$ converges to $y$, and $g(y)=g(1,1,1, \ldots)$ which we assume to be 1 .

On the other hand, $f(\alpha(t))=\exp (i t)$, and so $g((t))= \pm \exp (i t / 2)$, and again the sign cannot change. So $g(y)=-1$. Hence $f$ cannot have a continuous square root. A simple modification shows that it could not have a $m$ th root for any $m \geq 2$. We formulate this.

THEOREM 2. The space $H$ is the union of a nested sequence of compact sets $H_{n}$. Let $A_{n}=C\left(H_{n}, \mathrm{C}\right)$ be the continuous complex valued functions on $H_{n}$. Let $A=C(H, \Sigma, \mathbf{C})$ be the functions on $H$ which for each $n$ when restricted to $H_{n}$ fall into $A_{n}$. There is an $f$ in $A$ whose restriction to $H_{n}$ has a continuous nth root in $A_{n}$ for every $n$. But $f$ is not the nth power of any element of $A$ with $n$ greater than 1. 
3. The algebraic version of these theorems. We evidently have here an inverse system

$$
A_{1} \leftarrow A_{2} \leftarrow A_{3} \leftarrow \cdots
$$

of Banach algebra homomorphisms. This defines an inverse limit algebra $A$. This $A$ has an element which is not an $n$th power of any element of $A$ for any $n$ greater than 1 . However, its image $f_{n}$ in $A_{n}$ is an $n$th power of an element of $A_{n}$ for each $n$.

In our example, the algebras are commutative, and $f$ is invertible.

By way of contrast, in [1] it is shown that if $f_{n}$ has a right inverse in each $A_{n}$, then $f$ also has a right inverse in $A$.

4. The inverse limit of exponentials. This topic is not in the realm of pure algebra, but of topological algebra. We will show that the inverse limit of exponentials is not necessarily an exponential.

Let $X_{n}$ be the set of bounded complex sequences $\left(\lambda_{1}, \lambda_{2}, \lambda_{3}, \ldots\right)$ for which $\lambda_{m}$ is constant modulo $\mathbf{Z}$ from $n$ onward, with bound $B_{n} \leq n$.

Suppose there were a continuous $g$ such $g(x)$ that differs from each $g_{n}(x)$ by an integer at most. Then $g(x)=g(y)$ if $x$ and $y$ agree from some point on, because the obvious curve from $x$ to $y$ lies in some $X_{n}$. Let $\alpha(\theta)$ be $(\theta, \theta, \theta, \ldots)$. Then $g_{n}(\alpha(\theta))=\theta$. Therefore $g((\theta))=\theta+j, j$ an integer. This $j$ is constant. It suffices to treat the case where it is 0 . Then $g(0,0,0, \ldots)=0$ and $g(1,1,1, \ldots)=1$.

Let $x_{k}$ have $k$ zeros and then all 1's. These elements $x_{k}$ are all in $X_{1}$, and so is $(0,0,0, \ldots)$. The limit of the $x_{k}$ is $(0,0,0, \ldots)$. By the $x$ and $y$ argument, the $g\left(x_{k}\right)$ are all equal to 1 . Hence $g(0,0, \ldots)$ should be the limit of these 1's, and so be 1 .

This contradiction proves the following theorem, expressed in the notation of Theorem 2 .

THEOREM 3. The space $H$ is the union of a nested sequence of compact sets $H_{n}$. Let $A_{n}=C\left(H_{n}, \mathbf{C}\right)$ be the continuous complex valued functions on $H_{n}$. Let $A=C(H, \Sigma, \mathbf{C})$ be the functions on $H$ which for each $n$ when restricted to $H_{n}$ fall into $A_{n}$. There is an $f$ in $A$ whose restriction to $H_{n}$ has a continuous logarithm in $A_{n}$ for every $n$. But $f$ is not the exponential of any element of $A$.

Here we are taking $f(x)$ to be the common value of the numbers $\exp \left[2 \pi i g_{n}(x)\right]$ where $n$ is large enough so that $x \in X_{n}$. 


\section{REFERENCES}

[1] Richard Arens, Dense inverse limit rings, Michigan Math. J., 5 (1958), 169-182.

[2] R. Brown, Ten topologies for $X-Y$, Quart J. Math. Oxford (2), 14 (1963), 303-319.

Received March 2, 1988.

UNIVERSITY OF CALIFORNIA

LoS ANGELES, CA 90024-1555-05 


\section{PACIFIC JOURNAL OF MATHEMATICS EDITORS}

\author{
V. S. VARADARAJAN \\ (Managing Editor) \\ University of California \\ Los Angeles, CA 90024 \\ Herbert Clemens \\ University of Utah \\ Salt Lake City, UT 84112 \\ THOMAS ENRIGHT \\ University of California, San Diego \\ La Jolla, CA 92093
}

R. FINN

Stanford University

Stanford, CA 94305

HeRmanN FlaschKa

University of Arizona

Tucson, AZ 85721

VAUGHAN F. R. JONES

University of California

Berkeley, CA 94720

STEVEN KeRCKHOFF

Stanford University

Stanford, CA 94305
RoBION KIRBY

University of California

Berkeley, CA 94720

C. C. MOore

University of California

Berkeley, CA 94720

HAROLD STARK

University of California, San La Jolla, CA 92093

\section{ASSOCIATE EDITORS}
R. ARENS
E. F. BECKENBACH
B. H. NeUmanN
F. WolF
K. YosHIDA (1906-1982)

\section{SUPPORTING INSTITUTIONS}
UNIVERSITY OF ARIZONA
UNIVERSITY OF BRITISH COLUMBIA CALIFORNIA INSTITUTE OF TECHNOLOGY UNIVERSITY OF CALIFORNIA MONTANA STATE UNIVERSITY UNIVERSITY OF NEVADA, RENO NEW MEXICO STATE UNIVERSITY OREGON STATE UNIVERSITY
UNIVERSITY OF OREGON UNIVERSITY OF SOUTHERN CALIFORNIA STANFORD UNIVERSITY UNIVERSITY OF HAWAII UNIVERSITY OF TOKYO UNIVERSITY OF UTAH WASHINGTON STATE UNIVERSITY UNIVERSITY OF WASHINGTON 


\section{Pacific Journal of Mathematics}

\section{Vol. 137, No. $1 \quad$ January, 1989}

V. S. Varadarajan, Henry Abel Dye $\ldots \ldots \ldots \ldots \ldots \ldots \ldots \ldots \ldots \ldots$ iii

Huzihiro Araki, An application of Dye's theorem on projection lattices to orthogonally decomposable isomorphisms $\ldots \ldots \ldots \ldots \ldots \ldots \ldots \ldots \ldots$

Richard Arens, The limit of a sequence of squares in an algebra need not be

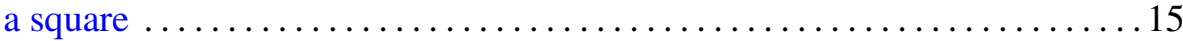

William Arveson, An addition formula for the index of semigroups of

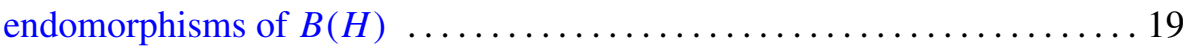

Robert James Blattner and Susan Montgomery, Crossed products and

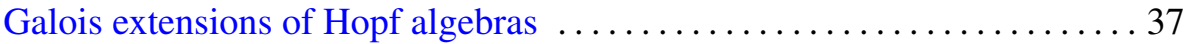

Erik Christensen and Allan M. Sinclair, On the vanishing of $H^{n}\left(\mathscr{A}, \mathscr{A}^{*}\right)$

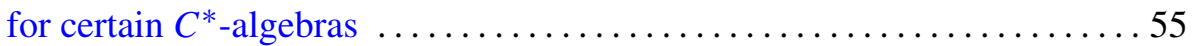

Philip C. Curtis, Jr. and Michael M. Neumann, Nonanalytic functional

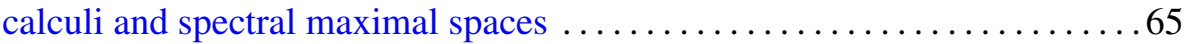

George A. Elliott and David E. Handelman, Addition of $C^{*}$-algebra extensions

Yaakov Friedman and Bernard Russo, Some affine geometric aspects of

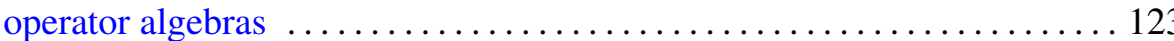

Valentin Ya. Golodets and Sergey D. Sinelshchikov, Regularization of actions of groups and groupoids on measured equivalence relations . . . 145

Irving Kaplansky, CCR-rings 155

Hideki Kosaki, Characterization of crossed product (properly infinite case)

Gert Kjærgaard Pedersen, Three quavers on unitary elements in $C^{*}$-algebras

Sorin Popa, Relative dimension, towers of projections and commuting squares of subfactors

Martin E. Walter, On a new method for defining the norm of

Fourier-Stieltjes algebras 